\title{
How Do We Make Clinical Molecular Testing for Cancer Standard of Care for Pathology Departments?
}

\author{
Sanam Loghavi, MD; Mark J. Routbort, MD, PhDa; Keyur P. Patel, MD, PhDª Rajyalakshmi Luthra, PhD; \\ Wei-Lien Wang, MD ; Russell R. Broaddus, MD, PhD ${ }^{\mathrm{b}}$; Michael A. Davies, MD, $\mathrm{PhD}^{\text {c }}$; and
}

Alexander J. Lazar, MD, $\mathrm{PhD}^{\mathrm{b}}$

\begin{abstract}
Understanding of the genetic basis and molecular pathogenesis of cancer has evolved substantially over the past century. The advent of high-throughput gene sequencing methods has unraveled hundreds of recurrent somatic genetic alterations in various malignancies, either causative or harboring major prognostic and/or predictive implications. Knowledge of these specific changes has dramatically altered diagnostic and therapeutic approaches to cancer, enabling personalized molecular therapies. This article shares approaches to adopting and fine-tuning the practice of molecular diagnostics as an essential component of diagnostic pathology in a tertiary care cancer hospital and proposes methods by which genetic testing in cancer can become standard of care in pathology departments across the nation.
\end{abstract}

J Natl Compr Canc Netw 2016;14(6):787-792

\section{Background}

Understanding of the genetic basis and molecular pathogenesis of cancer has evolved substantially over the past century. In 1914, Boveri ${ }^{1}$ first proposed that somatic genetic alterations were involved in the development of cancer. Approximately 50 years later, Nowell and Hungerford ${ }^{2}$ discovered the first recurrent chromosomal abnormality, the Philadelphia chromosome, in chronic myelogenous leukemia (CML). More recently, the advent of high-throughput gene sequencing methods (see supplemental eAppendix 1, available with this article at JNCCN.org) has unraveled hundreds of recurrent somatic genetic alterations in various malignancies, either causative or harboring major prognostic and/or predictive implications. Knowledge of these specific changes has dramatically altered the diagnostic and therapeutic approaches to cancer, enabling personalized molecular therapies. ${ }^{3-12}$

From the Departments of a Hematopathology, bPathology, and cMelanoma Medical Oncology, The University of Texas MD Anderson Cancer Center, Houston Texas.

Submitted January 11, 2016; accepted for publication March 8, 2016.
Somatic mutations play a substantial role in the diagnosis and prognostication of many solid and hematologic neoplasms. ${ }^{13-34}$ We now have strong evidence to support molecular testing in patients with various types of cancer, including lung carcinoma, melanoma, colorectal carcinoma, and acute myeloid leukemia, as part of routine clinical practice and standard of care, based on molecular markers for prognostic stratification and improved clinical outcomes with selective molecular therapy. ${ }^{13-20,22,23,27,28,31,33,35}$ Recent advances in sequencing technology and the ever-increasing availability and affordability of high-throughput sequencing platforms have facilitated the identification of relevant molecular targets. ${ }^{33,36-41}$ BRAF and MEK inhibitors for melanoma with BRAF p.V600E, EGFR inhibitors for RAS wild-type colorectal cancer, KIT inhibitors for patients with gastrointestinal stromal tumors with KIT or PDGFR mutations, and kinase inhibitors for acute

The authors have disclosed that they have no financial interests, arrangements, affiliations, or commercial interests with the manufacturers of any products discussed in this article or their competitors.

Correspondence: Alexander J. Lazar, MD, PhD, Department of Pathology, The University of Texas MD Anderson Cancer Center, 1515 Holcombe Boulevard, Unit 0085, Houston, TX 77030.

E-mail: alazar@mdanderson.org 
Loghavi et al

myeloid leukemia with FLT3 internal tandem duplication are a few examples of current targeted therapeutic agents. Before the discovery of tyrosine kinase inhibitors, patients with CML had a grim prognosis; in the current era of selective medicine, patients with CML have a survival rate comparable to the general population if appropriately treated. ${ }^{42,43}$ As such, molecular markers of disease are now an essential component of risk stratification and therapeutic planning for many patients with cancer.

In addition to prognostication and targeted therapy, it has been shown by several groups more recently that molecular alterations can determine response to immunotherapy. A few examples include melanoma in which loss of PTEN and activation of tumor intrinsic $\beta$-catenin signaling confer resistance to T-cellmediated immunotherapy and inferior outcomes with PD-1 inhibitor therapy and monoclonal antibodies against CTLA-4, respectively, ${ }^{44-46}$ and a subset of melanoma cases in which a specific genetic signature is linked to better response to ipilimumab. ${ }^{47}$ In addition, increasing total mutational load of melanoma is predictive of longer-term benefit with ipilimumab treatment, although the association trend is difficult to apply to individual patients due to overlapping ranges and needs further characterization. ${ }^{47,48}$

The pathology community is fortunate to be at the forefront of this revolution. This article shares approaches to adopting and fine-tuning the practice of molecular diagnostics as an essential component of diagnostic pathology in a tertiary care cancer hospital, and proposes methods by which genetic testing in cancer can become standard of care in pathology departments across the nation.

\section{What Do We Mean by "Making Molecular Testing Standard of Care"?}

Many laboratories currently lack the resources and infrastructure to perform complex molecular testing in-house. Development of comprehensive molecular profiling platforms may not be technically feasible, and doing so may not be a fiscally sound and/or viable option for many smaller laboratories; however, any laboratory that offers diagnostic pathology services for patients with cancer must have procedures in place to ensure appropriate procurement and processing of tumor tissues that will facilitate downstream molecular testing when necessary. A variety of suppliers of cancer molecular testing panels are available, but internal expertise must exist to determine the best patients and samples for testing and interpreting and delivering the results when an external supplier is used.

\section{Specimen Acquisition and Tissue Qualification}

The field of molecular medicine has generally advanced more rapidly in the context of myeloid neoplasia, due to the abundance of fresh material and readily obtainable blood and bone marrow samples that can be sorted using flow cytometry. Unfortunately, the same is not true for solid tumors, for which samples are often less generous in quantity and sometimes not clinically amenable to subsequent procurement when not secured upfront and in a timely fashion. Therefore, obtaining sufficient tumor tissue for downstream testing is of utmost priority. Fortunately, current DNA sequencing platforms, including nextgeneration sequencing (NGS), are well-suited for testing formalin-fixed paraffin-embedded tissue samples, which posed a major impediment historically.

The University of Texas MD Anderson Cancer Center has instituted a Tissue Qualification Laboratory (TQL) where patient pathology reports are reviewed by dedicated pathologists in order to select the best case and sample for requested molecular testing. Hematoxylin-eosin-stained sections of the tumor sample of interest and normal (non-neoplastic) tissue are reviewed and marked by an expert pathologist dedicated to the TQL service. This guides the manual microdissection that will facilitate enrichment of tumor content in subsequent DNA or RNA extraction. Tissue size and tumor percentage/ cellularity are used as general guidelines to determine whether further molecular testing, including NGS, can be performed. Tumor cellularity is an important preanalytical factor that influences the precision and accuracy of downstream sequencing. The sensitivity of the assay can be significantly compromised in samples with low percentage of tumor cells. Knowing the estimated tumor percentage can also facilitate the correct interpretation of sequencing results. As a general rule, we use 20\% involvement by tumor as a minimum cutoff to determine whether the tissue can be subjected to sequencing studies. This cutoff was established based on the usually heterozygous nature of somatic mutations and the lower limit of detection of the readily available sequencing platforms (approximately 5\%) in order to avoid tumor dilution and false-negative calls in the context of background noise. Examination of copy number variations in samples with low tumor percentages is particularly challenging. We generally resort to la- 
ser microdissection for highly precious samples and difficult-to-obtain samples with a tumor cellularity of less than $20 \%$.

Many platforms, more specifically those designed to detect fusion proteins, including those seen in lymphoid and myeloid malignancies, are designed to use RNA, and therefore upfront extraction of RNA is essential in order to permit downstream testing on these platforms.

\section{Available Molecular Testing Platforms}

Numerous molecular testing platforms are currently available both in CLIA-approved clinical laboratories and in research settings. DNA sequencing platforms include whole-genome sequencing (WGS); wholeexome sequencing (WES); multiplexed, targeted exome or hot-spot mutation panels for both solid and hematologic malignancies; and more traditional single-gene sequencing methods (eg, Sanger sequencing, pyrosequencing, capillary electrophoresis). Many laboratories use broader (either targeted exome or targeted hot-spot) panels. Although more information is usually of interest for research, the number of genes with validated clinical impact is relatively small. The ideal number of genes to include in a panel is evolving and influenced by both the needs and interests of the institution, technical needs and limitations, and certain financial realities. Having in-house capacity to perform this testing allows panels to be specifically adapted to institutional needs, although outsourcing is still a viable option. Single-gene analysis can still be useful for certain discrete decisions-a p.V600 mutation present in BRAF suggesting BRAF and MEK inhibitors-or to confirm findings on a larger panel when results are unclear.

Additional platforms interrogating RNA, micro RNA (miRNA), epigenomics, proteomics, and metabolomics are also available for use, although not as frequently and widely applied as DNA sequencing methods. However, RNA is relatively widely used and these other analytes will be increasingly important in clinical practice with time.

More recently, identification of circulating tumor biomarkers, including cell-free DNA and exosomes in the plasma of patients with various malignancies, has sparked much interest. These methods may provide avenues for serial follow-up of patients with solid tumors to determine response to therapy and for detection of early relapse, especially for tumors that may not be readily amenable to tissue biopsy. ${ }^{49-53}$ Screening for new cancer cases with these technologies could also have a role in high-risk populations.

\section{Data Analysis}

Recent advances in technology have resulted in relatively wide and inexpensive access to high-throughput sequencing methods. This advantage comes at the price of exponentially increasing raw data elements that need to be parsed and translated into clinically meaningful data via strategically designed bioinformatics pipelines. Most commercially available sequencing platforms are accompanied by at least a basic form of bioinformatic software; however, data analysis and interpretation continue to pose a significant challenge, even in the setting of major academic institutions with custom designed bioinformatic pipelines. Optimizing and validating pipelines is critical to avoid interlaboratory variability in variant (mutation) calling depending on the software and analytic algorithms used. ${ }^{54}$ In addition to known preanalytical factors (eg, specimen quality), the quality and reliability of a given assay is affected by several analytic and postanalytic factors, including depth of coverage, base quality (Phred quality or $Q$ score), and sequence alignment methods.

The lower limit of detection for most commercially available NGS platforms with adequate depth of coverage (at least 200 reads per base) is approximately 5\% mutant allele in the background of nonmutated DNA. Whether known driver mutations with very low frequencies in an adequately cellular tumor sample should be used to guide clinical decisionmaking is a subject of debate and raises important issues of tumor heterogeneity and clonal evolution (discussed further in the following section).

\section{Clonal Heterogeneity and Genomic Evolution}

Multiplexed NGS platforms can be used to study clonal heterogeneity within tumors to identify potentially resistant subclones in the setting of targeted therapy and various forms of clonal divergence and evolution that can give rise to subsequent metastasis and relapse. ${ }^{55,56}$ Although paired analysis of primary and metastatic lesions will yield more information with respect to tumor biology and clonal evolution, as a general approach, sequencing DNA of the most recently acquired metastatic lesion is preferred in terms of therapeutic planning, because this is presumably most similar to the tumor driving the clinical course of the patient being considered 
for systemic therapies. ${ }^{37,57,58}$ Although approximately $80 \%$ to $90 \%$ of primary and metastatic lesions show identical mutational profiles, a recent study from our group showed that in a smaller subset of cases (approximately 15\%) distinctive mutations may be seen in metastatic lesions, providing potential targets for therapy in patients with advanced disease. ${ }^{56}$ Some recent studies suggest that subclonal structure (eg, intratumoral heterogeneity) can affect cancer fitness, with too little or too much being associated with less aggressive behavior, whereas intermediate levels predict a worse outcome in multiple cancer types from The Cancer Genome Atlas (TCGA). ${ }^{59}$ Thus, assessment of subclonality structure could become more important with time, although obviously sampling issues strongly influence such applications.

\section{To Cast a Wide Net or Target the High Yield}

Because of the relatively low cost and wide availability of custom ("home-brewed") or commercially available multiplex sequencing panels, most institutions have elected to use multiplex platforms in lieu of single-gene testing as a way to identify actionably relevant gene mutations in a timely and cost-effective fashion. Generally, if there is a clinical interest in knowing the mutational or copy number status of at least 3 to 5 genes, it is more efficient to run these in the context of a more comprehensive panel than as single-gene tests that may require multiple polymerase chain reactions for proper coverage of each individual gene.

There are both advantages and disadvantages to using multiplex panels designed to capture large quantity of data in a single testing session. Although the relatively low cost, wide availability, and rather rapid nature of these tests render them highly attractive for use in routine clinical settings, the immensely large amount of data that is procured from these platforms is both an opportunity and a challenge. Our knowledge of the meaning and implications of many molecular alterations has not evolved as rapidly as technology has in this field. One the most challenging aspects of molecular diagnostics is determining the clinical implications of novel mutations and alterations of unknown clinical significance. Although various resources and public knowledge bases that keep record of specific mutation frequencies in various cancer types, including COSMIC (http://cancer.sanger. ac.uk/cosmic), dbSNP (http://www.ncbi.nlm.nih.gov/ SNP/), TCGA data portal (https://tcga-data.nci.nih. gov/tcga/), and cBioPortal (http://www.cbioportal. org/), are available and can be used for data interpretation and annotation, novel alterations are routinely discovered that have not been described previously in these resources and must be interpreted in the specific clinical context. ${ }^{60}$ Additional tools and computational software packages are available that aid in determining the functional impact of various somatic mutations, including SIFT and PolyPhen. ${ }^{61,62}$ Determining the type of mutation (silent vs nonsynonymous) and knowledge of the normal function of the affected gene can be helpful in these instances. For example, activating mutations of known oncogenes tend to be highly characteristic and recurrent, whereas inactivation, early termination, and dominant negative mutations in known tumor suppressor genes are highly suspected to be clinically relevant in the context of cancer, even if previously undescribed. Whether these novel mutations bear prognostic or predictive significance will be determined with time, although the rarity of certain mutations may practically preclude demonstration of effects in randomized clinical trials. Nevertheless, a multidisciplinary approach and clinicopathologic correlation are essential for accurate and meaningful interpretation of data in the appropriate clinical context.

As a further complication, some somatic testing laboratories do not use paired normal tissue for routine somatic mutation analysis. This poses an additional challenge of determining the nature (germline vs somatic) of some variants of unknown significance. Bioinformatic algorithms can be used to determine the likelihood of somatic status of mutations based on various factors, including percent depth of coverage, allele frequencies, and ratio of tumor to normal tissue in the analyzed sample. Nevertheless, germline mutations can be identified incidentally during somatic mutation testing and harbor specific clinical implications that need to be appropriately addressed. ${ }^{63,64}$ The approach of not using paired normal DNA from the patient is more practical in smaller, more focused panels, but complications still are regularly encountered that would be readily precluded by the use of paired normal DNA. The certainty provided by knowledge of germline status is accompanied by the cost of additional sequencing and the effort to acquire and process normal samples. 


\section{Annotation and Reporting}

Somatic mutations in genes shown to impact protein function, impart malignant behavior, be prognostically or predictively relevant or identified as targets of therapeutic agents, or known to confer resistance to therapy are classified as actionable or potentially actionable variants; any such alterations should be clearly stated in the report. ${ }^{37}$ Whether variants of uncertain significance should be included in the final pathology report is debatable, but many experts suggest that an inclusive approach is preferred with a notation to highlight the unknown clinical significance of these alterations. Various reporting schemes using different tiers of classification have been proposed based mostly on the degree of actionability of mutations..$^{65,66}$ No such system is universally accepted currently.

\section{Conclusions}

Providing molecular testing for every patient with cancer requires appropriate infrastructure that will allow real-time testing with technologies and assays that are currently available. Ever-evolving technologies also require thoughtful consideration as to how and when they should be incorporated into clinical practice for patient care and how they can support important institutional priorities for translational results that underlie future patient care. Vigorous and systematic efforts to permit banking of DNA and tissues indefinitely and the implementation of efficient and comprehensive technical and bioinformatic pipelines to enable data storage in massive quantities and reduce the cost of molecular testing are critical in this current environment of increasing fiscal restraint. It is also important to review and publish results demonstrating that molecular testing in cancer is not only cost-effective but also adds value to patient care by allowing more informed therapeutic choices. Establishing and using these methods require dedicated support from health care administrators and payors because the current reimbursement plans and options for molecular testing are less than favorable. This ultimately poses a major impediment to globalization of this revolutionary practice. There is a need to demonstrate that broader NGS provides benefit to patients and that it drives efficiencies for precision therapeutic selection in our health care delivery system. This would provide a strong basis for improved reimbursement.

The regulatory environment for clinical molecular testing, and in particular NGS, is evolving and care- ful attention must be paid to balancing the need for regulation to ensure quality and protect patients versus maintaining opportunities for innovation that can apply more rapidly emerging knowledge and technologies to benefit patients. Comprehensive studies using broad sequencing approaches are needed in the future to demonstrate the benefits and effectiveness of a broader approach in genetic testing. Despite the title of this article, we need to have patient protocols in place that help guide broad molecular testing where it can have impact and perhaps prevent testing in situations in which there is no or negligible benefit. Such strategies are hard to implement within our current health care environment, but discipline and restraint do have their place. Currently, many smaller practices, especially those based in the community setting, are using broad cancer molecular testing panels offered by commercial laboratories; we strongly advocate the need for internal expertise to determine the best patients and samples for testing and for interpreting and delivering the results in these instances.

Finally, we cannot emphasize enough the importance of a multidisciplinary approach, including input from colleagues in medical oncology, pathology, translational research, surgery, and radiation oncology, to promote a system that is beneficial to all parties involved, most importantly our patients.

\section{References}

1. Boveri T. Zur Frage der Entstehung maligner Tumoren. Jena, Germany: Gustav Fischer; 1914.

2. Nowell $\mathrm{P}$, Hungerford D. A minute chromosome in human chronic granulocytic leukemia. Science 1960;132:1497.

3. Genomic and epigenomic landscapes of adult de novo acute myeloid leukemia. N Engl J Med 2013;368:2059-2074.

4. Patel JP, Gonen M, Figueroa ME, et al. Prognostic relevance of integrated genetic profiling in acute myeloid leukemia. N Engl J Med 2012;366:1079_ 1089.

5. Cancer Genome Atlas Network. Genomic Classification of Cutaneous Melanoma. Cell 2015;161:1681-1696.

6. Barber TD, Vogelstein B, Kinzler KW, Velculescu VE. Somatic mutations of EGFR in colorectal cancers and glioblastomas. N Engl J Med 2004;351:2883.

7. Kobayashi S, Boggon TJ, Dayaram T, et al. EGFR mutation and resistance of non-small-cell lung cancer to gefitinib. N Engl J Med 2005;352:786-792.

8. Lassman AB, Abrey LE, Gilbert MR. Response of glioblastomas to EGFR kinase inhibitors. N Engl J Med 2006;354:525-526; author reply 526.

9. Messersmith WA, Ahnen DJ. Targeting EGFR in colorectal cancer. N Engl J Med 2008;359:1834-1836.

10. Gazdar AF. Personalized medicine and inhibition of EGFR signaling in lung cancer. N Engl J Med 2009;361:1018-1020.

11. He Y. Rociletinib in EGFR-mutated non-small-cell lung cancer. N Engl J Med 2015;373:578

12. Sequist LV, Rolfe L, Allen AR. Rociletinib in EGFR-mutated non-small-cell lung cancer. N Engl J Med 2015;373:578-579.

13. Bradish JR, Cheng L. Molecular pathology of malignant melanoma: changing the clinical practice paradigm toward a personalized approach. Hum Pathol 2014;45:1315-1326.

14. Cohen $R$, Cervera P, Svrcek $M$, et al. [DNA mismatch repair and BRAF status in colorectal cancer: Interest for the therapeutic management?]. Bull Cancer 2015;102(6 Suppl 1):S72-81. 


\section{Loghavi et al}

15. Cortes J, Calvo E, Vivancos A, et al. New approach to cancer therapy based on a molecularly defined cancer classification. CA Cancer J Clin 2014;64:70-74.

16. Dohner H, Estey EH, Amadori S, et al. Diagnosis and management of acute myeloid leukemia in adults: recommendations from an international expert panel, on behalf of the European LeukemiaNet. Blood 2010;115:453-474.

17. Harbst $K$, Staaf J, Lauss $M$, et al. Molecular profiling reveals low- and high-grade forms of primary melanoma. Clin Cancer Res 2012;18:4026-4036.

18. Kashani-Sabet M. Molecular markers in melanoma. $\mathrm{Br} J$ Dermatol 2014;170:31-35.

19. Tremante E, Ginebri A, Lo Monaco E, et al. Melanoma molecular classes and prognosis in the postgenomic era. Lancet Oncol 2012;13:e205-211.

20. Woodman SE, Lazar AJ, Aldape KD, Davies MA. New strategies in melanoma: molecular testing in advanced disease. Clin Cancer Res 2012;18:1195-1200.

21. Cancer Genome Atlas Network. Comprehensive molecular characterization of human colon and rectal cancer. Nature 2012;487:330-337.

22. Hamilton SR. Targeted therapy of cancer: new roles for pathologists in colorectal cancer. Mod Pathol 2008;21(Suppl 2):S23-30.

23. O'Donnell MR, Abboud CN, Altman J, et al. Acute myeloid leukemia. J Natl Compr Canc Netw 2012;10:984-1021.

24. Roth AD, Tejpar S, Delorenzi M, et al. Prognostic role of KRAS and BRAF in stage II and III resected colon cancer: results of the translational study on the PETACC-3, EORTC 40993, SAKK 60-00 trial. J Clin Oncol 2010;28:466474.

25. Van Cutsem E, Kohne CH, Lang I, et al. Cetuximab plus irinotecan, fluorouracil, and leucovorin as first-line treatment for metastatic colorectal cancer: updated analysis of overall survival according to tumor KRAS and BRAF mutation status. J Clin Oncol 2011;29:2011-2019.

26. Hutchins G, Southward K, Handley K, et al. Value of mismatch repair, KRAS, and BRAF mutations in predicting recurrence and benefits from chemotherapy in colorectal cancer. J Clin Oncol 2011;29:1261-1270.

27. Mrozek K, Marcucci G, Nicolet D, et al. Prognostic significance of the European LeukemiaNet standardized system for reporting cytogenetic and molecular alterations in adults with acute myeloid leukemia. J Clin Oncol 2012;30:4515-4523.

28. Artale $S$, Sartore-Bianchi A, Veronese SM, et al. Mutations of KRAS and BRAF in primary and matched metastatic sites of colorectal cancer. J Clin Oncol 2008;26:4217-4219.

29. Tol J, Nagtegaal ID, Punt CJ. BRAF mutation in metastatic colorectal cancer. N Engl J Med 2009;361:98-99.

30. Li Z, Herold T, He C, et al. Identification of a 24-gene prognostic signature that improves the European LeukemiaNet risk classification of acute myeloid leukemia: an international collaborative study. J Clin Oncol 2013;31:11721181 .

31. Andre T, de Gramont A, Vernerey D, et al. Adjuvant fluorouracil, leucovorin, and oxaliplatin in stage II to III colon cancer: updated 10-year survival and outcomes according to BRAF mutation and mismatch repair status of the MOSAIC Study. J Clin Oncol 201533:4176-4187.

32. Di Nicolantonio F, Martini M, Molinari F, et al. Wild-type BRAF is required for response to panitumumab or cetuximab in metastatic colorectal cancer. J Clin Oncol 2008;26:5705-5712.

33. Pietrantonio F, Petrelli F, Coinu A, et al. Predictive role of BRAF mutations in patients with advanced colorectal cancer receiving cetuximab and panitumumab: a meta-analysis. Eur J Cancer 2015;51:587-594.

34. Yoon $\mathrm{HH}$, Tougeron $\mathrm{D}$, Shi $\mathrm{Q}$, et al. KRAS codon 12 and 13 mutations in relation to disease-free survival in BRAF-wild-type stage III colon cancers from an adjuvant chemotherapy trial (N0147 alliance). Clin Cancer Res 2014;20:3033-3043.

35. Liu Y, Patel L, Mills GB, et al. Clinical significance of CTNNB1 mutation and Wnt pathway activation in endometrioid endometrial carcinoma. J Natl Cancer Inst 2014;106.

36. Siroy AE, Boland GM, Milton DR, et al. Beyond BRAF(V600): clinical mutation panel testing by next-generation sequencing in advanced melanoma. J Invest Dermatol 2015;135:508-515.

37. Meric-Bernstam F, Johnson A, Holla V, et al. A decision support framework for genomically informed investigational cancer therapy. J Natl Cancer Inst 2015;107.

38. Linnekamp JF, Wang X, Medema JP, Vermeulen L. Colorectal cancer heterogeneity and targeted therapy: a case for molecular disease subtypes. Cancer Res 2015;75:245-249.

39. Le Du F, Eckhardt BL, Lim B, et al. Is the future of personalized therapy in triplenegative breast cancer based on molecular subtype? Oncotarget 2015;6:1289012908.

40. Daver N, Cortes J, Ravandi F, et al. Secondary mutations as mediators of resistance to targeted therapy in leukemia. Blood 2015;125:3236-3245.

41. Cancer Genome Atlas Network. Genomic Classification of Cutaneous Melanoma. Cell 2015;161:1681-1696.
42. Mace ML, Dahl J, Jabbour EJ. Which tyrosine-kinase inhibitor to use first in chronic phase chronic myelogenous leukemia? Expert Opin Pharmacother 2015;16:999-1007.

43. Kantarjian $\mathrm{H}$, O'Brien $\mathrm{S}$, Jabbour E, et al. Improved survival in chronic myeloid leukemia since the introduction of imatinib therapy: a single-institution historical experience. Blood 2012;119:1981-1987.

44. Peng W, Chen JQ, Liu C, et al. Loss of PTEN promotes resistance to T cellmediated immunotherapy. Cancer Discov 2016;6:202-216.

45. Spranger S, Bao R, Gajewski TF. Melanoma-intrinsic beta-catenin signalling prevents anti-tumour immunity. Nature 2015;523:231-235.

46. Spranger S, Gajewski TF. A new paradigm for tumor immune escape: betacatenin-driven immune exclusion. J Immunother Cancer 2015;3:43

47. Snyder A, Makarov V, Merghoub T, et al. Genetic basis for clinical response to CTLA-4 blockade in melanoma. N Engl J Med 2014;371:2189-2199.

48. Van Allen EM, Miao D, Schilling B, et al. Genomic correlates of response to CTLA-4 blockade in metastatic melanoma. Science 2015;350:207-211.

49. Hyun KA, Kim J, Gwak H, Jung HI. Isolation and enrichment of circulating biomarkers for cancer screening, detection, and diagnostics. Analyst 2016;141:382-392.

50. Ravelli A, Reuben JM, Lanza F, et al. Breast cancer circulating biomarkers: advantages, drawbacks, and new insights. Tumour Biol 2015;36:6653-6665.

51. Alegre E, Sammamed M, Fernandez-Landazuri S, et al. Circulating biomarkers in malignant melanoma. Adv Clin Chem 2015;69:47-89.

52. Gold B, Cankovic M, Furtado LV, et al. Do circulating tumor cells, exosomes, and circulating tumor nucleic acids have clinical utility? A report of the association for molecular pathology. J Mol Diagn 2015;17:209-224.

53. Tabernero J, Lenz HJ, Siena S, et al. Analysis of circulating DNA and protein biomarkers to predict the clinical activity of regorafenib and assess prognosis in patients with metastatic colorectal cancer: a retrospective, exploratory analysis of the CORRECT trial. Lancet Oncol 2015;16:937-948.

54. O'Rawe J, Jiang $T$, Sun G, et al. Low concordance of multiple variant-calling pipelines: practical implications for exome and genome sequencing. Genome Med 2013;5:28.

55. Ding L, Ley TJ, Larson DE, et al. Clonal evolution in relapsed acute myeloid leukaemia revealed by whole-genome sequencing. Nature 2012;481:506-510.

56. Goswami RS, Patel KP, Singh RR, et al. Hotspot mutation panel testing reveals clonal evolution in a study of 265 paired primary and metastatic tumors. Clin Cancer Res 2015;21:2644-2651.

57. Meric-Bernstam F, Farhangfar C, Mendelsohn J, Mills GB. Building a personalized medicine infrastructure at a major cancer center. J Clin Oncol 2013;31:1849-1857.

58. Meric-Bernstam F, Mills GB. Overcoming implementation challenges of personalized cancer therapy. Nat Rev Clin Oncol 2012;9:542-548.

59. Andor N, Graham TA, Jansen M, et al. Pan-cancer analysis of the extent and consequences of intratumor heterogeneity. Nat Med 2016;22:105-113.

60. Bamford S, Dawson E, Forbes S, et al. The COSMIC (Catalogue of Somatic Mutations in Cancer) database and website. Br J Cancer 2004;91:355-358.

61. Kumar P, Henikoff S, Ng PC. Predicting the effects of coding non-synonymous variants on protein function using the SIFT algorithm. Nat Protoc 2009;4:1073-1081.

62. Adzhubei IA, Schmidt S, Peshkin L, et al. A method and server for predicting damaging missense mutations. Nat Methods 2010;7:248-249.

63. Green RC, Berg JS, Grody WW, et al. ACMG recommendations for reporting of incidental findings in clinical exome and genome sequencing. Genet Med 2013;15:556-574.

64. Parsons DW, Roy A, Plon SE, et al. Clinical tumor sequencing: an incidental casualty of the American College of Medical Genetics and Genomics recommendations for reporting of incidental findings. J Clin Oncol 2014;32:2203-2205.

65. MacConaill LE, Garcia E, Shivdasani P, et al. Prospective enterprise-level molecular genotyping of a cohort of cancer patients. J Mol Diagn 2014;16:660672.

66. Andre F, Mardis E, Salm M, et al. Prioritizing targets for precision cancer medicine. Ann Oncol 2014;25:2295-2303. 